\title{
Cloud Computing for brain segmentation technology
}

\author{
Victor Chang \\ School of Computing, Creative Technologies and Engineering, Leeds Metropolitan University, Leeds, UK \\ Electronics and Computer Science, University of Southampton, Southampton, UK \\ V.I.Chang@leedsmet.ac.uk
}

\begin{abstract}
This paper introduces the brain segmentation technology offered by Cloud Computing. It explains eleven APIs associated with each brain segment, as well as the process of capturing data in regard to each segment. Functionality and experiments associated with each API are discussed. Dancing is chosen because data related to fast and skilled movements can be captured more easily. The results captured for each brain segment are discussed and used to explain why some segments are more active in dancing. With an emphasis in testing to ensure a high quality of data analysis and visualization, eleven Cloud APIs can produce results quickly, accurately and effectively. Simulations for brain segmentations can be used by Medical Cloud Computing Education (MCCE). Results of analysis confirms that Cloud Computing can offer $20 \%$ improvement in learning satisfaction. Benefits of using Cloud brain segmentation technology are presented. The use of Cloud Computing can make positive impacts to healthcare informatics and education.
\end{abstract}

Keywords- Healthcare Cloud; brain segmentation technology by Cloud Computing; Medical Cloud Computing Education.

\section{INTRODUCTION}

Cloud Computing is reported to offer added values for organizational adoption in service computing and IT service management, where both Education and Healthcare sectors have demonstrated proof-of-concepts for delivering the next generations of scientific research, business models and education $[1,2,3,4,5,6,7,8,9]$. Healthcare informatics plays a strategic role in the UK National Health Service (NHS) to improve the level of healthcare services and the quality of education and research. These NHS IT initiatives include Cloud Computing, which demonstrates that scientific work can be enhanced; collaboration can be improved and IT services can be delivered. Fusion between healthcare, education and Cloud Computing can provide greater benefits to scientists, medical students and healthcare staff and successful fusion becomes increasingly important in modern healthcare development $[4,10,11]$. Hence, more universities have used Cloud Computing for education and research with case studies supported by NHS, University of Oxford and King's College London (KCL) $[4,9,12]$. There are interests to develop medical Cloud Computing including brain segmentation [13]. Medical Cloud Computing can thus offer inter-disciplinary collaboration in investigating (a) learning satisfaction and brain response and (b) learning difficulties and brain response.

The advancement in technologies is a driving force to blend with medical education and research $[10,13]$. Together with improvements in learning strategies, Cloud Computing technologies can influence the way medical education and research can move forward $[13,14,15]$. To fulfill this longterm vision, this paper presents an innovative approach for blending both medical education and research, where brain segmentation is a case study to illustrate its positive impacts. The objective is to study how human brain responds while recapturing a skill that was learned two years ago. The research was conducted in a motivated and interactive environment to study the positive impacts offered by Cloud computing. Data collections for volunteers are conducted and analyzed for discussions. The breakdown for this paper is as follows. Section 2 describes the structure of human brain and explains the importance to understand this prior discussing Cloud Computing technologies and evaluation. Section 3 presents Cloud Computing system design and equipment used for preliminary experiments and approaches with regard to brain segmentation. Section 4 presents the results of actual experiments in the form of brain segmentation visualization. Section 5 presents four related topics for discussions. Section 6 sums up conclusion and future work.

\section{STRUCTURE OF THE BRAIN}

Understanding structures and functions of the human brain will be useful to see the connections between brain segmentation and Cloud Computing. The structure of the human brain is in Figure 1 as follows.

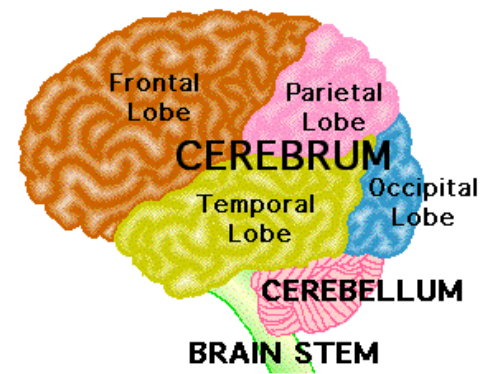

Figure 1: The simplified structure of the human brain

The structure and function of the human brain is explained as follows:

- Frontal Lobe: It controls behaviors, intelligence, memory (mainly short term) and movement.

- Parietal Lobe: It controls intelligence, language, reading and sensation.

- Temporal lobe: it controls behavior, hearing, storing new memory, speech and vision.

- Occipital Lobe: it processes human vision.

- Cerebellum: It controls balance and co-ordination. 
- Brain stem: It controls blood pressure, breathing, consciousness, heartbeat and swallowing.

Each part of the human brain has its own functions and roles. Although some parts have overlapped functions, they have specific roles and responsibility for the human body. One example is memory, where frontal lobe is responsible for short term memory and temporal lobe is responsible for storing new memory. Long term memory will be stored in the inner layers of the frontal lobe.

\section{A. How does this relate to our study?}

Activities in our human brain are reflected in our learning. There are several research questions to explore including the relationship between intelligence and brain activities; how learning can stimulate our brain and vice versa; how to develop each learner's potential through learning; how to maximize learning while understanding neuroscience more [16, 17, 18]. This motivates us to have a more established link between learning and brain science. While taking up new skills, our brain cells react differently which is reflected as follows. Some people can learn a new skill quickly and also remembers it for a long time. Some people need more time to learn a new skill, and also have a high tendency to lose the skill if it is not practiced for some time. The majority of people need a while to learn a new skill and can still retain their skill for a different of time (dependent on individuals).There are researchers who interpret that all these observations are due to the direct link between intelligence and memory. Those with high Intelligence Quotient (IQ) can learn faster and remember longer [18, 19, 20]. Some researchers disagree and argue that this is towards the attitudes of learning that affect activities of the brain [16, $17,21]$. Although this question is not a classic "chicken and egg", it is important that scientists and educationist understand how people learn and how our brain cells react, and understand brain activities in the process of learning.

\section{B. Movement for capturing brain activities}

It is more challenging to record brain activities for a sedative skill that requires demonstrations in intellectual abilities. On the other hand, it is much easier to record brain activities that involve volunteers with physical movement. The reason is their heart beat and emotion will change apart from their brain activities. In this way, brain waves and associated activities can be collected more easily than a sedative activity that requires a high level of concentration for some time.

Dancing is chosen as it involves with rapid but sophisticated movement, and lots of movements can produce sufficient data for brain activities in different parts of the brain. Two groups of volunteers with eight in each group took part the study. Each of them attached a "brain-wave detection" device while taking part in dancing. Brain waves were recorded and sent to a private cloud which stored all the data and analyzed them. Cloud Application Programming Interfaces (APIs) are used to process and analyze data and present volunteers' brain activities (as a collective result) on brain segmentation. Details will be presented in Section 4.

\section{CLOUD SEGMENTATION - SYSTEM DESIGNS AND PRELIMINARY EXPERIMENTS}

This section describes contributions from Cloud Computing for this project. Descriptions including Cloud Computing software and approaches and preliminary work before the actual experiments are provided.

\section{A. System Design of Cloud APIs}

Application Programming Interfaces (APIs) are used in Cloud applications with the following advantages. Firstly, scientific complexity such as numerous mathematical formulas associated with neuroscience need not to be presented up front, and this can improves the usability and interactions between researchers and volunteers during the experiments. Researches need not always process data or fix operational problems (such as system administration issues, or restart of software programs) during experiments. Secondly, APIs only need to be presented with an interface that can receive data, process data and present data, including both numeric results and visualization. This can reduce the time to perform additional computational work during experiments. There are five Cloud APIs described as follows.

- UpperFrontLobe - This API is to read the data from "brain wave device" attached to each volunteer, and it is wirelessly connected to the nearest private cloud server (wireless speed: 100 Mbps). The APIs connected to the device record the quantity and intensity of data. This API will compute a numeric number, as 0.000 as the lowest and 1.000 as the highest. If 1.000 is presented, the entire upper region of frontal lobe of human brain will be filled with visualization, which represents the overall activities of brain cells.

- LowerFrontLobe - Similar to above, except it is responsible for the lower front lobe.

- UpperParietalLobe - Same as above, except it is responsible for the upper parietal lobe.

- LowerParietalLobe - Same as above, except it is responsible for the lower parietal lobe.

- OccipitalLobe - There is only one API for Occipital Lobe as it is mainly dealing with human vision.

- LeftTemporalLobe - The functionality is the same as the first four APIs, except it is for left side of Temporal Lobe. Due to the structural difference, Temporal Lobe is divided into the left and right for APIs.

- $\quad$ RightTemporalLobe - Same as above, except it is for right side of Temporal Lobe.

- LeftCerebellum - Same as above, except it is for left side of Cerebellum.

- RightCerebellum - Same as above, except it is for right side of Cerebellum.

- UpperBrainStem - The functionality is the same as the previous APIs, except it is for the upper side of Brain Stem. 
- LowerBrainStem - Same as above, except it is for the lower side of Brain Stem.

\section{B. Algorithms involved}

Each Cloud API has common funcitons explained as follow:

- $\quad$ read (data) - This function reads all numerical data from "brain-wave device".

- $\quad$ process (data) - This function processes the data from the read operation. Collected data is recorded as datasets, which can be varied dependent on the quantity of the input data.

- update (data) - This function update all the data values for read (data) and process (data).

- visualize (data) - this function represents the numerical values in the phase of process (data). The intensity of each brain segment is represented by the numerical values collected and calculated.

Every second all the datasets are read and processed. Organizational Sustainability Modeling (OSM) is used to process the data $[7,13]$. The detail will not be presented here due to the shortage of length and avoidance of sideline the focus of this paper. In summary, OSM can process datasets efficiently and quickly. For example, if each "brain-wave device" had collected 500 datasets, OSM read all values and sums them, average them out (normally with an averaging ratio of 1:5), and displays the results. All the collected data received multiple of 5 of datasets, and can go to the upper most limit of 10,000 . For the purpose of the experiments, 4,000 datasets were collected and analyzed, with an averaging ratio of 10. Matrix A contains all collected datasets. An example for code can be presented in Table 1as follows.

Table 1: Average execution time for different Cloud APIs

for $(\mathrm{i}==0 ; \mathrm{i}<=400 ; \mathrm{i}++) / / 4000$ divides 10 is equal to 400
read (Matrix A);
process (Matrix A);
update (Matrix A);
visualize (Matrix A);
end;

\section{Preliminary work}

Before investigating the brain cell activities in brain segmentation, preliminary work is required for two reasons. Firstly, it aims to test whether the connection and process between "brain-wave device" and "Cloud APIs" can work fine. Secondly, it helps researchers identify the execution time required to process data and present data in visualization. Preliminary work involved with a pair of volunteers dancing, and most of dancing moves were salsa. Each volunteer had attached the "brain-wave device". In another preliminary test, it was confirmed that the device did not affect the quality of data recorded in the brain wave device. Preliminary tests were conducted so that each API could process datasets and had their execution time recorded. Execution time on five occasions was taken to get an average execution time with their standard deviations. Results are presented in Table 2.
Table 2: Average execution time for different Cloud APIs

\begin{tabular}{|l|l|l|}
\hline Cloud APIs & $\begin{array}{l}\text { Average execution } \\
\text { time }(\mathrm{sec})\end{array}$ & $\begin{array}{l}\text { Standard } \\
\text { deviation }(\mathrm{sec})\end{array}$ \\
\hline UpperFrontLobe & 5.28 & 0.06 \\
\hline LowerFrontLobe & 5.16 & 0.05 \\
\hline UpperParietalLobe & 5.23 & 0.06 \\
\hline LowerParietalLobe & 5.14 & 0.05 \\
\hline OccipitalLobe & 4.86 & 0.05 \\
\hline LeftTemporalLobe & 4.35 & 0.05 \\
\hline RightTemporalLobe & 4.46 & 0.05 \\
\hline LeftCerebellum & 4.23 & 0.04 \\
\hline RightCerebellum & 4.31 & 0.05 \\
\hline UpperBrainStem & 4.18 & 0.04 \\
\hline LowerBrainStem & 4.09 & 0.04 \\
\hline
\end{tabular}

Results in Table 2 show that all the average execution time is completed between 4.09 and 5.28 seconds, with standard deviations of between 0.04 and 0.06 seconds. The reason why some APIs take slightly longer time is that the time to record data from each region to the device and to data processing is dependent on the position of the brain segmentation. Dancing is an activity that requires a lot of body movement and coordination. Thus, brain stem and cerebellum, the most relevant regions in the brain segmentation, are most likely to be filled in datasets and be completed the data processing and visualization the quickest. Whereas regions away from brain stem, are less likely to be filled with datasets [22]. Data processing is thus the mostly likely to be completed at the last. Low standard deviations suggest that collected datasets and data processing with read, process and visualize steps (described in Section 4 B) are consistent with each other.

\section{Cloud SEGMENTATION - ACTUAL EXPERIMENTS AND BRAIN SEGMENTATION}

This section describes the actual experiments and its results in execution time and visualization. Different tests were undertaken to ensure that Cloud APIs can produce results quickly, accurately and effectively.

\section{A. Actual Experiments}

As discussed in Section 2B, eight pairs of volunteers took part and their brain response waves were recorded and then processed by Cloud APIs. All of them had dancing experience but stopped dancing for at least two years. The objective is to understand how they could recapture their skills, and the respective brain activities (reflected in segmentation). Execution time on five occasions was taken to get an average execution time with their standard deviations. Results are presented in Table 3.

Comparing to Table 2, results in Table 3 show that there is overall shorter average execution time. The most sensible explanation is that in a larger dancing group, many volunteers were very delighted, with very happy movement and shouting of joy all the times. As a result, it was quicker to fill the device with the required number of datasets. There is an average between 0.20 and 0.24 seconds quicker across all APIs. However, standard deviations widen due to a higher number of 
volunteers involved, but the difference between 0.07 and 0.11 is an acceptable range. Average execution time in the last four APIs are between 0.22 and 0.24 seconds shorter than the counterparts in Table 2, which may mean that it is quicker to be filled with datasets and be ready for processing. On the other hand, average execution time in the first four APIs still take longer time than the average of all APIs due to the distance and less relevance to dancing.

Table 3: Average execution time for different Cloud APIs

\begin{tabular}{|l|l|l|}
\hline Cloud APIs & $\begin{array}{l}\text { Average execution } \\
\text { time }(\mathrm{sec})\end{array}$ & $\begin{array}{l}\text { Standard } \\
\text { deviation }(\mathrm{sec})\end{array}$ \\
\hline UpperFrontLobe & 4.98 & 0.11 \\
\hline LowerFrontLobe & 4.86 & 0.10 \\
\hline UpperParietalLobe & 4.95 & 0.10 \\
\hline LowerParietalLobe & 4.81 & 0.10 \\
\hline OccipitalLobe & 4.64 & 0.10 \\
\hline LeftTemporalLobe & 4.14 & 0.09 \\
\hline RightTemporalLobe & 4.25 & 0.09 \\
\hline LeftCerebellum & 4.01 & 0.08 \\
\hline RightCerebellum & 4.07 & 0.08 \\
\hline UpperBrainStem & 3.92 & 0.07 \\
\hline LowerBrainStem & 3.85 & 0.07 \\
\hline
\end{tabular}

\section{B. Brain segmenetation}

As discussed in Section 2, the objective is to study how volunteers respond when they reuse a skill, which they learned at least two years ago but did not practice before the start of experiment. When all the datasets are processed, each API computes a numerical value corresponding to the intensity of each region in brain segmentation. Visualization is the end result of such representation. Figure 2 shows the diagram the collective result of brain activities while recapturing dancing skills. It shows different levels of brain activities in the frontal lobe, parietal lobe, occipital lobe (these three are grey matters, the last one is situated at the back), temporal lobe consisting of corpus callosum and hippocampus (inner brain, the later one controls emotion) and brain stem (middle brain).

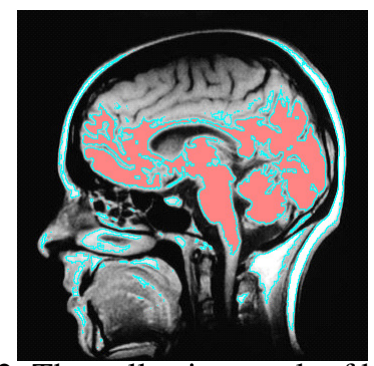

Figure 2: The collective result of brain activities while recapturing dancing skills

The result shows that brain activities in the lower frontal lobe, lower parietal lobe and occipital lobe are reacting actively. The entire brain stem and temporal lobe are responding very positively and the intensity level is at the highest. This may mean volunteers require a high level of balancing. When they dance, their movement is fast, and must keep themselves balanced with swift but steady movements. The areas that represent the emotions are not so obvious but still can be seen with some activities. Experimental results also agree with existing theories [16, 17, 18, 19, 20, 21, 22].

\section{Numerical values for the intensity level}

This section presents numerical values corresponding to Figure 2, where Table 4 shows the numerical results of the volunteer's collective brain intensity.

Table 4: Average execution time for different Cloud APIs

\begin{tabular}{|l|l|l|}
\hline Cloud APIs & $\begin{array}{l}\text { Average numerical } \\
\text { value (out of 1) }\end{array}$ & $\begin{array}{l}\text { Standard } \\
\text { deviation }\end{array}$ \\
\hline UpperFrontLobe & 0.024 & 0.010 \\
\hline LowerFrontLobe & 0.705 & 0.111 \\
\hline UpperParietalLobe & 0.022 & 0.009 \\
\hline LowerParietalLobe & 0.717 & 0.115 \\
\hline OccipitalLobe & 0.991 & 0.009 \\
\hline LeftTemporalLobe & 0.915 & 0.025 \\
\hline RightTemporalLobe & 0.919 & 0.025 \\
\hline LeftCerebellum & 0.986 & 0.008 \\
\hline RightCerebellum & 0.990 & 0.015 \\
\hline UpperBrainStem & 1.000 & 0.001 \\
\hline LowerBrainStem & 1.000 & 0.001 \\
\hline
\end{tabular}

APIs with high numerical values mean that they have high brain activities during the experiments. Explanations for APIs above 0.90 are as follows.

- Occipital Lobe - The ability for vision is enhanced because eyes need to capture various dancing movements in a short period of time.

- $\quad$ LeftTemporalLobe - The ability for the control all of behaviors, hearing, storing new memory and vision is improved and can react to fast changes in dancing.

- RightTemporalLobe - Same as LeftTemporalLobe except it has higher values. A likely reason is that dancing is considered as arts and the right side of the brain processes most of interpretations related to arts than the left side of the brain.

- LeftCerebellum - The ability to control balance and co-ordination is greatly enhanced due to fast and skilled movements required by dancing.

- RightCerebellum - Same as LeftCerebellum except it has a marginally higher value. A likely reason is that right side of the brain processes more interpretations than the left side.

- UpperBrainstem and LowerBrainstem - both have a numeric value equal to 1.000 , the highest value recorded. This means that in dancing, human body reacts fully to control blood pressure, breathing, consciousness and heartbeat to ensure that dancers can be fully ready for dancing movements.

\section{DISCUSSIONS: BENEFITS OF USING CLOUD COMPUTING}

This section describes the benefits of using Cloud Computing for scientific research. Topics of discussions include reproducibility and reusability of results; cost-savings; benefits to education and future research questions. 


\section{A. Reproducibility and reusability}

Reproducibility is an important aspect in Science that similar results should be reproducible while following steps and recommendations from the previous experiment [23]. Experiments involved with physical and biological science have this characteristic in common to ensure results are reproducible when working in the same scientific conditions. Apart from reproducibility, reusability is important. Since large-scale experiments can take a lot of funding and time for planning and execution, it is desirable that part of, or the entire set up or results of any scientific can be reused.

Cloud computing for brain segmentation technology can meet reproducibility and reusability described as follows. Firstly, results of experiments in Section 4 fully agree with the theories. Brain segments that are closely related to movement, co-ordination, behaviors, memory, hearing, vision, breathing, heartbeat, blood pressure and consciousness have hyper activities. Secondly, each experiment was conducted five times to get the average result. Each time results for each of ten brain segments have similar outcomes with a small standard deviation ranging from 0.001 and 0.111 . In the case of reusability, the most expensive part was the technical set up of the experiments. Once the set up and experiments were completed, results were recorded in the Cloud Computing brain segmentation technology service. Data can be used to represent the collective results of the investigation without purchasing or renting the equipment to set up. This can save several thousands pound for set up fee each time. Results can be computed in the form of numerical values or $3 \mathrm{D}$ simulations showing the activities of each brain segment in regard to dancing. Cloud computing simulation can be reused at any time without the set up and results of the collective effort can be used to analyze the impact between learning and brain activities [13].

\section{B. Cost-savings}

Similar research can take years to get comparable results and they may cost millions of British pounds. Often the organization that receive funding are Cancer Research UK or Neurophysiology. At the NHS, the emphasis is on medicine as they offer NHS Practitioner Training Program. The exact expenditure is not enclosed to the public. However, the estimated cost can be analyzed briefly as follows. The project can cost multiple millions to offer training, and another multiple millions to provide facilities and another few years of training and millions of investment are required in order to produce similar results as this research paper demonstrates. In contrast, the facility for this research cost $£ 81,000$ set up, and another $£ 2,000$ for loan of equipment. The volunteers and collaboration with University of London Computing Center (ULCC) and KCL are free of charge.

According to NHS statement, the NHS will spend $£ 110$ billion in 2015/2016 year, a $0.1 \%$ increase of current spending [24]. While searching for various references and public reports, NHS does not give an exact figure on IT spending. Based on the author's previous experience working with the NHS, $1 \%$ of the budget spending on its national IT application and infrastructure (such as Electronic Patients' Records) is perhaps a good estimate. Assuming $1 \%$ of $£ 110$ billions in spent in the NHS, an estimated 1.1 billion pounds of IT spending is used annually. However, the NHS efficiency including IT services are criticized by the public [25]. In some cases, millions of pounds are spent without delivering services. Or three times more than the original budget is required to deliver. Some IT projects above $£ 1$ million do not support reproducibility and repeatability in contrast to this project. Altogether the cost of this project is only $£ 83,000$ excluding the extra time and effort, which fully meets the tight budget. This amount of spending between 2009 and 2011 is only $0.754 \%$ of the estimated spending by the NHS UK in a year. This project can demonstrate a high extent of cost-saving if the right level of skills and competency are implemented for the healthcare informatics, without the need to apply for extra funding and extended delivery deadline.

\section{The benefits to education}

Chang [13] presents an evaluation and statistical analysis of two medical cohort groups based on the implementation of this brain segmentation technology. The results confirm that Medical Cloud Computing Education" (MCCE) can improve $20 \%$ of learning satisfaction. Two medical cohorts show more interests and acknowledgment in the use of Cloud Computing blending with medical education. Statistical results confirm a good extent of accuracy in analysis by having high R-squared values; low root mean square values and high F-test values. The variations in p-value have been explained by the variations before and after using Cloud Computing for education. Based on direct feedback from some participants, MCCE can enhance learning and understanding of complex science such as brain segmentation.

Similarly, Cloud Computing can be used for education to produce similar outcomes. There are case studies described as follows. Firstly, the University of Southampton adopted Cloud Computing. It was reported to offer added values for two departments, Electronics and Computer Science (ECS) and the i-Solution Group, where cost-saving exceeded 20\% for ECS and service improvement had around 4 to $8 \%$ comparing to the previous years $[6,15]$. Secondly, the University of Greenwich used Cloud Computing to deliver supply chain courses. It was reported to have $15 \%$ improvement in learning satisfaction and students could achieve higher academic performance of up to $15 \%$ while using Cloud Computing for education. Lecturers could save efforts to repeat same lectures over the period of time due to the use of simulations to simplify explanations of complex supply chain concepts [10]. Thirdly, it was reported that King's College London, NHS and University of Oxford adopted Cloud Computing and could offer better quality of teaching and IT services for stakeholders, scientists and students [4, 9, 11, 12, 13]. The use of Cloud Computing is crucial in the curriculum development and IT services at these universities based in the UK.

\section{Future research questions to investigate the relationship between brain segment and learning}

The relationship between how brain activities respond and learning can be aided by brain segmentation technology. This 
should include investigations to understand how people learn. Research questions include the following: "When each volunteer learn a new theory, how their brains react and which segments are the most active?"; "Does each person vary different in different parts of the brain segments while learning the same thing?"; "Does people from different ethic background react differently in their brain segments while learning the same thing?"; "Are there any ways to stimulate learning process and produce better learning efficiency?". With the understanding with brain segmentation, it can help more people to understand between learning and brain activities. These include how to improve our learning outcome when we can learn new skill, and also when we recapture a skill that was not practice for some time. The use of Cloud Computing can help us produce simulations to understand the process of learning.

\section{CONCLUSION AND FUTURE WORK}

This paper introduces the brain segmentation technology offered by Cloud Computing. It explains the APIs associated with each brain segment, as well as the process of capturing data in regard to each segment. Dancing is chosen because data related to fast and skilled movements can be captured more easily. The results captured for each brain segment are discussed, and they agree with the theories that some segments are more active in dancing. The average execution time for each API is presented and all analysis can be completed in seconds. Furthermore, an evaluation was used by Medical Cloud Computing Education" (MCCE). Results of analysis confirm that Cloud Computing can offer 20\% improvement in learning satisfaction. Benefits of using Cloud Computing with brain segmentation technology are presented. Reproducibility and reusability are supported. Cost-savings can be achieved, as the expenditure in 3 years is only $0.754 \%$ of the estimated spending by the NHS UK in a year. Benefits to education are illustrated by this case study, and with successful deliveries at the Universities of Southampton, Greenwich and Oxford and also KCL and NHS. Plan for the future work is as follows. More studies involved with body movements such as dancing will be investigated and more sample sizes will be included. Future collaboration with more research institutes will be strengthened to jointly deliver more funding and publication. The use of Cloud Computing can make positive impacts to healthcare informatics and education.

\section{REFERENCES}

[1] V. Chang, D. Bacigalupo, G. Wills and D. De Roure, "A Categorisation of Cloud Computing Business Models," In CCGrid 2010, The 10th IEEE/ACM International Symposium on Cluster, Cloud and Grid Computing, May 17-20, Melbourne, Australia, pp. 509-512.

[2] V. Chang, G. Wills and D. De Roure, "A Review of Cloud Business Models and Sustainability," In IEEE Cloud 2010, the third International Conference on Cloud Computing, 5-10 July, 2010, Miami, USA.

[3] Mohammed, A. B., Altmannn, J. and Hwang, J., “"Cloud Computing Value Chains: Understanding Businesses and Value Creation in the Cloud", special topic: Economic Models and Algorithms for Distributed Systems, Autonomic Systems, 2010, Part III, 187-208.

[4] V. Chang, D. De Roure, G. Wills and R. Walters, "Organisational Sustainability Modelling for Return on Investment: Case Studies presented by a National Health Service (NHS) Trust UK". Journal of Computing and Information Technology, 19 (3). ISSN Print ISSN 13301136 | Online ISSN 1846-3908.
[5] S. Marston, Z. Li, S. Bandyopadhyay, J., Zhang and A., Ghalsasi, "Cloud computing - The business perspective", Decision Support Systems, 51 (1), Publisher: Elsevier B.V., Pages: 176-189, 2011.

[6] V. Chang, R. Walters and G. Wills, "Business Integration as a Service," International Journal of Cloud Applications and Computing, 2 (1), 1640.

[7] V. Chang, "Business integration as a service: computational risk analysis for small and medium enterprises adopting SAP," International Journal of Next-Generation Computing, 4, (3) (In Press).

[8] V. Chang, D. De Roure, G. Wills and R. Walters, "Case Studies and Organisational Sustainability Modelling presented by Cloud Computing Business Framework," International Journal of Web Services Research. ISSN 1545-7362.

[9] V. Chang, R. Walters and G. Wills, "The development that leads to the Cloud Computing Business Framework," International Journal of Information Management, 33 (3), June 2013, pp. 524-538.

[10] V. Chang and G. Wills, "A University of Greenwich Case Study of Cloud Computing - Education as a Service," E-Logistics and E-Supply Chain Management: Applications for Evolving Business, IGI Global, April 2013.

[11] L.M. Vaquero, "EduCloud: PaaS versus IaaS Cloud Usage for an Advanced Computer Science Course", IEEE Transactions on Education archive, 4(4), pp. 590-598, November 2011.

[12] V. Chang, R. Walters and G. Wills, "Cloud Storage and Bioinformatics in a private cloud deployment: Lessons for Data Intensive research. In, Cloud Computing and Service Science, Springer Lecture Notes Series, Springer Book, September 2013.

[13] V. Chang, "Brain Segmentation - A Case study of Biomedical Cloud Computing for Education and Research," In Learning Technologies Workshop, Higher Education Academy (HEA), University of Greenwich, June 2013.

[14] A.L. Ostrom, M.J. Bitner, S.W. Brown, K.A. Burkhard, M. Goul, V. Smith-Daniels, H. Demirkan and E. Rabinovich, "Moving Forward and Making a Difference: Research Priorities for the Science of Service", Journal of Service Research; 13 (4), Janaury 2010.

[15] V. Chang, G. Wills, R. Walters and W. Currie, "Towards a structured Cloud ROI: The University of Southampton cost-saving and user satisfaction case studies," Sustainable Green Computing: Practices, Methodologies and Technologies, 2012, pp. 179-200.

[16] M.D. Kickmeier-Rust, D. Schwarz, D. Albert, D. Verpoorten, J-L. Castaigne, and M. Bopp, "The ELEKTRA project: Towards a new learning experience," M3 - Interdisciplinary aspects on digital media \& education (pp. 19-48).

[17] M. Maniruzzaman, "Learning EFL by Bengali Speaking Learners: Major Linguistic problems and possible solutions," Druck und Bindung, ISBN 978-3-640-64430-8, Germany, 2010.

[18] Mehta, M., "Behavioural Sciences in Medical Practice," Jaypee Bro Medical Publisher, ISBN 81-7179-582, 1998.

[19] K. Carter and C. Seifert, "Learn Psychology", published by Jones \& Bartlett Learning, ISBN 978-443-5000, 2013.

[20] M.D. Kickmeier-Rust, D. Schwarz, D. Albert, D. Verpoorten, J-L. Castaigne, and M. Bopp, "The ELEKTRA project: Towards a new learning experience," M3 - Interdisciplinary aspects on digital media \& education (pp. 19-48).

[21] V. Klucharev, A. Smidts and G. Fernández, "Brain mechanisms of persuasion: how 'expert power' modulates memory and attitudes", Soc Cogn Affect Neuroscience, 3(4): 353-366, December 2008.

[22] J.C. Fernandez-Miranda, S. Pathak, J. Engh, K. Jarbo, T. Verstynen, F.C Yeh, Y. Wang, A. Mintz, F. Boada, W. Schneider and R. Friedlander, "High-Definition Fiber Tractography of the Human Brain: Neuroanatomical Validation and Neurosurgical Applications", NeuroSurgery, 71 (2), August 2012.

[23] O. Dalle, "On Reproducibility and Tracibility of Simulaitons", in Winter Simulation Conference, 2012.

[24] J. Ousbey, "Spending Round 2013, Analysis and Response: NHS Confederation", white paper, NHS Confederation, June 2013.

[25] P. Turner, R. Kane and C Jackson, "Creating enterprise efficiencies in the NHS," British Journal of Healthcare Management, Vol. 19, Iss. 7, 24 Jul 2013, pp 330 - 334. 